\title{
45. Projective Parameters on Paths in D. van Dantzig's Projective Space.
}

\author{
By Kentaro YANO. \\ Mathematical Institute, Tokyo Imperial University. \\ (Comm. by S. KAKEYA, M.I.A., April 12, 1944.)
}

1. J. Haantjes ${ }^{1)}$ discussed a few years ago the projective geometry of paths with the use of D. van Dantzig's homogeneous curvilinear coordinates $^{2}$. In a generalized projective space $H_{n}$ referred to a system of van Dantzig's homogeneous curvilinear coordinate $\left(x^{\lambda}\right)^{3}$, if we introduce a projetive connection $\Pi_{\mu \nu}^{\lambda}$ satisfying the three conditions: (i) $\left(\partial \Pi_{\mu \nu}^{\lambda} / \partial x^{\omega}\right) x^{\omega}=-\Pi_{\mu \nu}^{\lambda}$, (ii) $\Pi_{\mu \nu}^{\lambda}=\Pi_{\nu \mu}^{\lambda}$ and (iii) $\Pi_{\mu \nu}^{\lambda} x^{\mu}=0$, the equations of paths or of autoparallel curves are

$$
\frac{d^{2} x^{\lambda}}{d r^{2}}+\Pi_{\mu \nu}^{\lambda} \frac{d x^{\mu}}{d r} \frac{d x^{\nu}}{d r}=\alpha \frac{d x^{\lambda}}{d r}+\beta x^{\lambda} .
$$

The finite equations of a path being $x^{\lambda}(r)$, Haantjes introduces two homogeneous coordinates $u^{a}$ on each path. Then the equations of paths may also be written as

$$
x^{\lambda}=x^{\lambda}\left(u^{a}\right),
$$

where the $x^{\lambda}\left(u^{a}\right)$ are homogeneous functions of $u^{a}$ of degree 1 , and the differential equations of paths take the form

$$
\frac{\partial^{2} x^{\lambda}}{\partial u^{b} \partial u^{c}}+\Pi_{\mu \nu}^{\lambda} \frac{\partial x^{\mu}}{\partial u^{b}} \frac{\partial x^{\nu}}{\partial u^{c}}=\Gamma_{b c}^{a} \frac{\partial x^{\lambda}}{\partial u^{a}} .
$$

The functions $\Gamma_{b o}^{a}$ appearing in (1.3) transform like the coefficients of a projective connection in an $H_{1}$, and satisfy the same conditions as (i), (ii) and (iii). Then, J. Haantjes proves that the curvature tensor formed with the $\Gamma_{b c}^{a}$ vanishes identically and therefore there exists a coordinate system $\left(u^{a}\right)$ for which $\Gamma_{b c}^{a}$ are all zero. This special coordinate system being determined up to linear homogeneous transformations with constant coefficients, the non homogeneous parameter

$$
t=u^{1} / u^{0}
$$

is determined up to linear fractional transformations. Thus, $t$ is a projective parameter defined on each path. J. Haantjes proves then

1) J. Haantjes: On the projective geometry of paths, Proc. Edinburgh Math. Soc. 5 (1937), 103-115.

2) D. van Dantzig: Theorie des projektiven Zusammenhangs $n$-dimensionaler Räume, Math. Ann. 106 (1932), 400-454; J. A. Schouten and J. Haantjes : Zur allgemeinen projektiven Differentialgeometrie, Compositio Math. 3 (1935), 1-51.

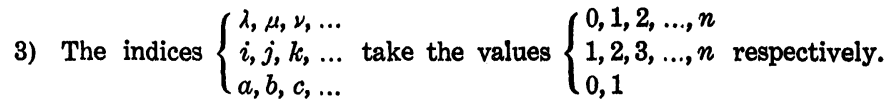


that, in the case of so-called normal projective connection, $t$ coincides with the L. Berwald's preferred projective parameter ${ }^{1)}$.

In previous papers ${ }^{2}$, the present author remarked that choosing a suitable function $\rho$ and a suitable parameter $t$, we can write the differential equations (1.1) in the form

$$
\frac{d^{2} \rho x^{\lambda}}{d t^{2}}+\Pi_{\mu \nu}^{\lambda}(\rho x) \frac{d \rho x^{\mu}}{d t} \frac{d \rho x^{\nu}}{d t}=0,
$$

and the parameter $t$ in (1.5) is a projective parameter. In the present Note, we will show that the parameter $t$ coincides with that of $\mathrm{L}$. Berwald not only in the case of normal projective connection, but also in the case of general projective connection.

2. To compare J. Haantjes' theory with L. Berwald's one or with the author's one, we must introduce the non homogeneous coordinate system $\left(\xi^{i}\right)$ in $H_{n}$. The non homogeneous coordinate system $\left(\xi^{i}\right)$ may be defined by

$$
\xi^{i}=\xi^{i}\left(x^{0}, x^{1}, x^{2}, \ldots, x^{n}\right),
$$

where $\xi^{i}(x)$ are homogeneous functions of degree zero, and hence

$$
E_{\cdot \lambda}^{i} x^{\lambda}=0 \text {, where } E_{\cdot \lambda}^{i}=\xi_{, \lambda}^{i}=\partial \xi^{i} / \partial x^{\lambda},
$$

the rank of the matrix $\left(E_{\cdot_{\lambda}}^{i}\right)$ being $n$.

To define the inverse of $\left(E_{\cdot}^{i}{ }_{\lambda}\right)$, we introduce a covariant projective vector $p_{\lambda}$ satisfying

$$
p_{\lambda} x^{\lambda}=1,
$$

and being the homogeneous functions of $x^{\lambda}$ of degree -1 .

Then the inverse $\left(E_{j}^{{ }^{\lambda}}\right)$ of $\left(E_{\cdot{ }_{\lambda}}^{i}\right)$ is defined by means of the equations

$$
E_{i j}^{\cdot \lambda} E_{\cdot \lambda}^{i}=\delta_{j}^{i} \text { and } E_{j}^{\cdot \lambda} p_{\lambda}=0 .
$$
lations

It will be easily proved that the $\left(E_{i}^{i}\right)$ and $\left(E_{\cdot{ }_{\lambda}}^{i}\right)$ satisfy the re-

$$
E_{\nu}^{\lambda} \equiv E_{i}^{\cdot \lambda} E_{\cdot{ }_{\nu}}^{i}=\delta_{\nu}^{\lambda}-x^{\lambda} p_{\nu} .
$$

The $\left(E_{\cdot_{\lambda}}^{i}\right),\left(E_{i}^{i^{\lambda}}\right)$ and $E_{\nu}^{\lambda}$ being thus defined, we introduce the quantities $\Gamma_{i k}^{0}$ and $\Gamma_{j k}^{i}$ by means of the equations

and

$$
\Gamma_{j k}^{0}=-E_{j}^{\mu} E_{k^{\nu}}^{\nu}\left(p_{\mu, \nu}-p_{\lambda} I I_{\mu \nu}^{\lambda}\right),
$$

$$
\Gamma_{j k}^{i}=-E_{j}^{\mu \mu} E_{k}^{\cdot \nu}\left(E_{\cdot \mu_{\nu},}^{i}-E_{\cdot{ }_{\lambda}}^{i} I I_{\mu \nu}^{\lambda}\right)
$$

respectively. It will be proved in the next paragraph that $\Gamma_{j k}^{0}$ are components of a tensor which defines the projective parameters on

1) L. Berwald: On the projective geometry of paths, Annals of Math. 37 (1936), 879-898.

2) K. Yano: Les espaces à connexion projective et la géométrie projective des paths, Annales Scientifiques de l'Université de Jassy, 24 (1938), 395-464; Projective parameters in projective and conformal geometries, Proc. 20 (1944), 45-53. 
paths and $\Gamma_{j k}^{i}$ are the coefficients of an affine connection which gives the same system of paths as the projective connection $\Pi_{\mu \nu}^{\lambda}$.

The choice of the covariant projective vector $p_{\lambda}$ being quite arbitrary but for the condition (2.3), we can take

$$
\bar{p}_{\lambda}=p_{\lambda}+\varphi_{\lambda}
$$

instead of $p_{\lambda}$. The $\bar{p}_{\lambda}$ and $p_{\lambda}$ are both covariant projective vector fields satisfying (2.3), the $\varphi_{\lambda}$ is also a covariant projective vector field satisfying

$$
\varphi_{\lambda} \bar{x}^{\lambda}=0 \text {. }
$$

If we effect the transformation of $p_{\lambda}$ of the type (2.8), the matrix $\left(E_{\cdot_{\lambda}}^{i}\right)$ is invariant, say, $\bar{E}_{\lambda}^{i}=E_{\cdot \lambda}^{i}$ but $\left(E_{j}^{*^{\lambda}}\right)$ is changed into $\left(\bar{E}_{j}^{\cdot \lambda}\right)$ following the formulae

$$
\bar{E}_{j}^{\cdot \lambda}=E_{j}^{\cdot \lambda}-x^{\lambda} \varphi_{j},
$$

where

(2.11) $\varphi_{j}=E_{j}^{*^{\lambda}} \varphi_{\lambda}$ and consequently by (2.5) and (2.9) $\varphi_{\lambda}=E_{\cdot \lambda}^{i} \varphi_{i}$.

Denoting by $\bar{\Gamma}_{j k}^{0}$ and $\bar{\Gamma}_{j k}^{i}$ the functions (2.6) and (2.7) defined by the $\bar{E}_{\cdot \lambda}^{i}$ and $\bar{E}_{j}^{* \lambda}$ respectively, we have

$$
\bar{\Gamma}_{j k}^{0}=\Gamma_{j k}^{0}-E_{j}^{\cdot \mu} E_{k}^{\cdot \nu}\left(\varphi_{\mu, \nu}-\varphi_{\lambda} I_{\mu \nu}^{\lambda}\right)-\varphi_{j} \varphi_{k}
$$

and

$$
\bar{\Gamma}_{j k}^{i}=\Gamma_{j k}^{i}-\delta_{j}^{i} \varphi_{k}-\delta_{k}^{i} \varphi_{j},
$$

by virtue of the relations

$$
x^{\mu} p_{\mu, \nu}+p_{\nu}=0, \quad x^{\nu} p_{\mu, \nu}+p_{\mu}=0, \quad x^{\mu} \varphi_{\mu, \nu}+\varphi_{\nu}=0 \text { and } x^{\nu} \varphi_{\mu, \nu}+\varphi_{\mu}=0
$$

obtained from (2.3), (2.9) and the fact that $p_{\lambda}$ and $\varphi_{\lambda}$ are both homogeneous functions of $x^{\lambda}$ of degree -1 .

On the other hand, differentiating the equations $\varphi_{\mu}=E_{{ }_{\mu}^{j}}^{j} \varphi_{j}$. with respect to $x^{\nu}$, we have

from which

$$
\varphi_{\mu, \nu}=E_{\cdot{ }_{\mu, \nu}, \varphi_{i}}^{i}+E_{. \mu}^{j} E_{{ }^{\prime}}^{k} \varphi_{j, k}, \quad\left(\varphi_{j, k}=\partial \varphi_{j} / \partial \xi^{k}\right)
$$

$$
E_{j}^{\cdot \mu} E_{k}^{\cdot \nu}\left(\varphi_{\mu, \nu}-\varphi_{\lambda} I_{\mu \nu}^{\lambda}\right)=\varphi_{j, k}-\varphi_{i} \Gamma_{j k}^{i}
$$

by virtue of (2.7) and (2.11). Thus, the equations (2.12) may also be written as

$$
\bar{\Gamma}_{j k}^{0}=\Gamma_{j k}^{0}-\varphi_{j, k}+\varphi_{i} \Gamma_{j k}^{i}-\varphi_{j} \varphi_{k} .
$$

The equations (2.13) and (2.15) show that the functions $\Gamma_{j k}^{0}$ and $\Gamma_{j k}^{i}$ are the coefficients of a projective connection studied by the American School' ${ }^{1)}$ and consequently, as the present author showed"), of É. Cartan's projective connection.

1) See, for example, O. Veblen: Projektive Relativitätstheorie, Berlin, Springer, 1933.

2) K. Yano: Les espaces a connexion projective et la géométrie projective des paths, loc. cit. 
We observe that the coefficients $\Pi_{\mu \nu}^{\lambda}$ of the projective connection being symmetric, the functions $\Gamma_{j k}^{i}$ defined by (2.7) are symmetric with respect to two indices $j$ and $k$, but the functions $\Gamma_{j k}^{0}$ defined by (2.6) are not necessarily symmetric with respect to two lower indices.

3. In this paragraph, we consider the equations of paths (1.1) which may be written in the form (1.5). If the equations (1.5) are expanded, we have

$$
\frac{d^{2} x^{\lambda}}{d t^{2}}+I I_{\mu \nu}^{\lambda} \frac{d x^{\mu}}{d t} \frac{d x^{\nu}}{d t}+\frac{2}{\rho} \frac{d \rho}{d t} \frac{d x^{\lambda}}{d t}+\frac{1}{\rho} \frac{d^{2} \rho}{d t^{2}} x^{\lambda}=0
$$

by virtue of the conditions $\Pi_{\mu \nu}^{\lambda} x^{\mu}=\Pi_{\mu \nu}^{\lambda} x^{\nu}=0$ and $\Pi_{\mu \nu}^{\lambda}(\rho x)=\rho^{-1} \Pi_{\mu \nu}^{\lambda}(x)$.

A solution of (3.1) being $x^{\lambda}(t)$, if we substitute this in (2.1), we have $\xi^{i}(t)$. We shall calculate the differential equations satisfied by these functions $\xi^{i}(t)$. Differentiating (2.1) with respect to $t$, we have successively

$$
\begin{aligned}
& \frac{d \xi^{i}}{d t}=E_{\cdot \lambda}^{i} \frac{d x^{\lambda}}{d t}, \\
& \frac{d^{2} \xi^{i}}{d t^{2}}=E_{\cdot \mu, \nu}^{i} \frac{d x^{\mu}}{d t} \frac{d x^{\nu}}{d t}+E_{\cdot \lambda}^{i} \frac{d^{2} x^{\lambda}}{d t^{2}},
\end{aligned}
$$

and hence, remembering (2.7), we have

$$
\frac{d^{2} \xi^{i}}{d t^{2}}+\Gamma_{j k}^{i} \frac{d \xi^{j}}{d t} \frac{d \xi^{l c}}{d t}=E_{\cdot \lambda}^{i}\left(\frac{d^{2} x^{\lambda}}{d t^{2}}+\Pi_{\mu \nu}^{\lambda} \frac{d x^{\mu}}{d t} \frac{d x^{\nu}}{d t}\right)-2 p_{\mu} \frac{d x^{\mu}}{d t} \frac{d \xi^{i}}{d t}
$$

by virtue of the relations

$$
E_{\nu}^{\lambda}=E_{i}^{\cdot \lambda} E_{\cdot{ }_{\nu}}^{i}=\delta_{\nu}^{\lambda}-x^{\lambda} p_{\nu}, \quad \Pi_{\mu \nu}^{\lambda} x^{\mu}=0, \quad E_{\cdot \lambda}^{i} \frac{d x^{\lambda}}{d t}=\frac{d \xi^{i}}{d t}
$$

and

$$
E_{\mu}^{\sigma} E_{\nu}^{\tau} E_{\cdot \sigma, \tau}^{i}=E_{\cdot \mu, \nu}^{i}+p_{\mu} E_{\cdot \nu}^{i}+p_{\nu} E_{\cdot \mu}^{i} .
$$

Substituting (3.1) in (3.2), we find

$$
\frac{d^{2} \xi^{i}}{d t^{2}}+\Gamma_{j k}^{i} \frac{d \xi^{j}}{d t} \frac{d \xi^{k}}{d t}+2\left(\frac{1}{\rho} \frac{d \rho}{d t}+p_{\mu} \frac{d x^{\mu}}{d t}\right) \frac{d \xi^{i}}{d t}=0 .
$$

Introducing here a parameter $s$ by the equations

$$
2\left(\frac{1}{\rho} \cdot \frac{d \rho}{d t}+p_{\mu} \frac{d x^{\mu}}{d t}\right)=\frac{d^{2} t}{d s^{2}} /\left(\frac{d t}{d s}\right)^{2},
$$

the above equations are written in the form

$$
\frac{d^{2} \xi^{i}}{d s^{2}}+\Gamma_{j k}^{i} \frac{d \xi^{j}}{d s} \frac{d \xi^{k}}{d s}=0
$$

which shows that the functions defined by (2.7) are coefficients of an affine connection which gives the same systcm of paths as the projective connection $\Pi_{\mu \nu}^{\lambda}$. The differential equations of paths in non homogeneous coordinates being thus obtained, we shall consider the relation between the parameters $t$ and s. Differentiating (3.3) with respect to $t$, we have 


$$
\begin{gathered}
2\left[-\frac{1}{\rho^{2}}\left(\frac{d \rho}{d t}\right)^{2}+\frac{1}{\rho} \frac{d^{2} \rho}{d t^{2}}+\left(p_{\mu, \nu}-p_{\lambda} \Pi_{\mu \nu}^{\lambda}\right) \frac{d x^{\mu}}{d t} \frac{d x^{\nu}}{d t}+p_{\lambda}\left(\frac{d^{2} x^{\lambda}}{d t^{2}}+\Pi_{\mu \nu}^{\lambda} \frac{d x^{\mu}}{d t} \frac{d x^{\nu}}{d t}\right)\right] \\
=\frac{d^{3} t}{d s^{3}} /\left(\frac{d t}{d s}\right)^{3}-2\left(\frac{d^{2} t}{d s^{2}}\right)^{2} /\left(\frac{d t}{d s}\right)^{4} .
\end{gathered}
$$

Substituting (3.1) in the above equation, we obtain

$$
\begin{gathered}
-\frac{2}{\rho^{2}}\left(\frac{d \rho}{d t}\right)^{2}- \\
-\frac{4}{\rho} \frac{d \rho}{d t} p_{\mu} \frac{d x^{\mu}}{d t}+2\left(p_{\mu, \nu}-p_{\lambda} \Pi_{\mu \nu}^{\lambda}\right) \frac{d x^{\mu}}{d t} \frac{d x^{\nu}}{d t} \\
=\frac{d^{3} t}{d s^{3}} /\left(\frac{d t}{d s}\right)^{3}-2\left(\frac{d^{2} t}{d s^{2}}\right)^{2} /\left(\frac{d t}{d s}\right)^{4} .
\end{gathered}
$$

The equations (3.3) and (3.5) give us

$$
\begin{aligned}
\{t, s\} & \equiv \frac{d^{3} t}{d s^{3}} / \frac{d t}{d s}-\frac{3}{2}\left(\frac{d^{2} t}{d s^{2}}\right)^{2} /\left(\frac{d t}{d s}\right)^{2} \\
& =2\left(p_{\mu, \nu}-p_{\lambda} \Pi_{\mu \nu}^{\lambda}+p_{\mu} p_{\nu}\right) \frac{d x^{\mu}}{d s} \frac{d x^{\nu}}{d s} .
\end{aligned}
$$

On the other hand, we have from (2.6)

$$
p_{\mu, \nu}-p_{\lambda} \Pi_{\mu \nu}^{\lambda}+p_{\mu} p_{\nu}=-E_{. \mu}^{j} E_{. \nu}^{k} \Gamma_{j k}^{0} .
$$

Substituting this in (3.6) we have finally

$$
\{t, s\}=-2 \Gamma_{j k}^{0} \frac{d \xi^{j}}{d s} \frac{d \xi^{k}}{d s} .
$$

Thus, the parameter $t$ being defined by a Schwarzian derivative, the $t$ is a projective parameter.

4. The equations (2.7) can also be written in the form

$$
E_{{ }_{\mu, \nu}}^{i}=E_{{ }_{\cdot}}^{i} \Pi_{\mu \nu}^{\lambda}-E^{j}{ }_{\mu} E_{{ }^{\prime}}^{k} \Gamma_{j k}^{i}-p_{\mu} E_{{ }_{\nu}}^{i}-p_{\nu} E_{{ }^{\prime}}^{i} .
$$

Expressing the integrability conditions of these equations, we find

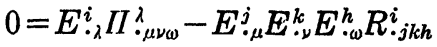

$$
\begin{aligned}
& +\left(p_{\mu, \nu}-p_{\lambda} I I_{\mu \nu}^{\lambda}+p_{\mu} p_{\nu}\right) E_{{ }^{\prime}}^{i}-\left(p_{\mu, \omega}-p_{\lambda} I I_{\mu \omega}^{\lambda}+p_{\mu} p_{\omega}\right) E_{{ }^{\prime}}^{i} \\
& -E_{\cdot \mu}^{i} p_{\nu, \omega}+E_{\cdot \mu}^{\imath} p_{\omega, \nu},
\end{aligned}
$$

where $I I_{\cdot \mu \nu \omega}^{\lambda}$ and $R_{\cdot j k h}^{\imath}$ are curvature tensors formed with $\Pi_{\mu \nu}^{\lambda}$ and $\Gamma_{j k}^{i}$ respectively. It is well known that the curvature tensor $I I_{\mu \nu \omega}^{\lambda}$ satisfies

$$
I I_{. \mu \nu \omega}^{\lambda} x^{\mu}=\Pi_{. \mu \nu \omega}^{\lambda} x^{\nu}=I \lambda_{{ }_{\mu \nu \omega}}^{\lambda} x^{\omega}=0 .
$$

The equations (4.2) can also be written as

$$
\text { (4.3) } \quad E_{{ }_{\lambda}}^{i} E_{j}^{\cdot \mu} E_{k}^{\cdot \nu} E_{h}^{\cdot \omega} \Pi_{{ }_{\mu \nu \omega}}^{\lambda}=R_{\cdot j k h}^{i}+\Gamma_{j k}^{0} \delta_{h}^{i}-\Gamma_{j h}^{0} \delta_{k}^{i}-\delta_{j}^{i}\left(\Gamma_{k h}^{0}-\Gamma_{h k}^{0}\right) \text {. }
$$

If the projective curvature is normal, that is to say, if the projective curvature tensor $I I_{\cdot \mu \nu \omega}^{\lambda}$ satisfies the condition $I I_{{ }_{\mu \nu \lambda}}^{\lambda}=0$, we have from (4.3), by contraction 
No. 4.] Projective Parameters on raths in D. van Dantzig's Projective Space.

$$
0=R_{j k}+n \Gamma_{j k}^{0}-\Gamma_{k j}^{0} \text {, }
$$

where $R_{j k}$ is the contracted curvature tensor $R_{\cdot j k i}^{i}$.

From (4.4) we have

$$
\Gamma_{j k}^{0}=-\frac{n R_{j k}+R_{k j}}{n^{2}-1}
$$

Substituting these values of $\Gamma_{j k}^{0}$ in (4.3), we have

(4.6) $E_{\cdot{ }_{2}}^{i} E_{j}^{\cdot \mu} E_{k}^{\cdot \nu} E_{h}^{\cdot \omega} \Pi_{\mu \nu \omega}^{\lambda}$

$$
\begin{aligned}
& =R_{\cdot j k h}^{i}-\frac{1}{n^{2}-1}\left(n R_{j k}+R_{k j}\right) \delta_{h}^{i}+\frac{1}{n^{2}-1}\left(n R_{j h}+R_{h j}\right) \delta_{k}^{i} \\
& +\frac{1}{n+1} \delta_{j}^{i}\left(R_{k h}-R_{h k}\right)
\end{aligned}
$$

which shows that $E_{\cdot{ }_{\lambda}}^{i} E_{j}^{\cdot \mu} E_{k}^{\cdot \nu} E_{h}^{\cdot \omega} \Pi_{{ }^{\prime}{ }_{\mu \nu \omega}}$ is the Weyl projective curvature tensor.

Substituting (4.5) in (3.7), we have

$$
\{t, s\}=\frac{2}{n-1} R_{j k} \frac{d \xi^{j}}{d s} \frac{d \xi^{k}}{d s} .
$$

Thus, our projective parameter $t$ coincides with what was discussed by L. Berwald and J. Haantjes. 